\title{
Stability Analysis of Micropolar Fluid Flow over an Exponentially Permeable Shrinking Sheet
}

\author{
Rusya Iryanti Yahaya ${ }^{1}$, Norihan Md Arifin ${ }^{1,2^{*}}$ and Siti Suzilliana Putri Mohamed Isa ${ }^{1,3}$ \\ ${ }^{1}$ Institute for Mathematical Research, Universiti Putra Malaysia, 43400 UPM Serdang Selangor, \\ Malaysia \\ ${ }^{2}$ Department of Mathematics, Universiti Putra Malaysia, 43400 UPM Serdang Selangor, Malaysia \\ ${ }^{3}$ Centre of Foundation Studies for Agricultural Science, Universiti Putra Malaysia, 43400 UPM \\ Serdang Selangor, Malaysia
}

\begin{abstract}
In the present paper, stability analysis is performed to the dual solutions obtained in boundary layer flow of micropolar fluid over a shrinking sheet with exponential velocity. The problem is first considered as time-dependent problem. Then, the governing equations are transformed into ordinary differential equations using similarity transformations. Linear eigenvalue equations are introduced, and the smallest eigenvalues are computed by using a MATLAB solver called the bvp4c solver. The first solution is found to have positive smallest eigenvalues, while the second solution has negative smallest eigenvalues. Thus, the stable solution is the first solution, while the second solution is unstable.
\end{abstract}

Keywords: stability analysis, dual solutions, micropolar fluid, shrinking sheet

\section{INTRODUCTION}

Micropolar fluid is a non-Newtonian fluid introduced by Eringen (1966). The fluid consists of colloidal fluid elements suspended in small body fluid. Therefore, the fluid has micro-rotational effect and micro-rotational inertia.

The boundary layer flow of micropolar fluid over shrinking surface was studied by Yacob \& Ishak (2012). This study was then extended by Bhattacharyya et al. (2012) with thermal radiation. Later, Roşca and Pop (2014) studied the flow with second-order slip velocity. Sharma et al. (2016) extended this study for another type of flow called the stagnation-point flow. Then, Zaimi and Ishak (2014) studied this flow over a non-linear stretching and shrinking sheet. Sandeep and Sulochana (2015) discussed the unsteady mixed convection flow of magneto-micropolar fluid. Aurangzaib et. al., (2016) then extended the study with partial slip and stagnation-point flow. On the other hand, the fluid flow over an exponentially shrinking sheet was studied by Aurangzaib et. al., (2016). The timedependent flow past a curved surface was discussed by
Saleh et. al., (2017). Khan et al. (2017) discussed the magnetohydrodynamics flow of the fluid with weak concentration. In all these studies, dual solutions were obtained.

Stability analysis of dual solutions is usually done to identify the stable solution which is significant to the problem. One of the earliest studies on stability analysis was done by Merkin (1986). The analysis was performed to the dual solutions obtained in the problem of mixed convection in a porous medium. In the study, the solutions were divided into the upper branch and the lower branch. It was concluded that the upper branch is stable while the lower branch is unstable. In the study of micropolar fluid flow with dual solutions, the same conclusion was obtained by Roşca \& Pop (2014) and Sharma et. al., (2016).

In the present study, the stability analysis is done for the dual solutions obtained by Aurangzaib et. al., (2016). This analysis is performed to identify the solution that is stable and significant to the problem. The computations will be done by using bvp4c solver. 


\section{PROBLEM FORMULATION}

A steady, incompressible two-dimensional boundary layer flow and heat transfer of micropolar fluid over a permeable shrinking sheet is considered. The sheet is shrunk with velocity $U_{w}=a e^{\frac{x}{L}}$ where $a>0$ is the shrinking constant.

The governing equations for the problem are:

$$
\begin{array}{r}
\frac{\partial u}{\partial x}+\frac{\partial v}{\partial y}=0, \\
u \frac{\partial u}{\partial x}+v \frac{\partial u}{\partial y}=\left(v+\frac{\kappa}{\rho}\right) \frac{\partial^{2} u}{\partial y^{2}}+\frac{\kappa}{\rho} \frac{\partial N}{\partial y} \\
u \frac{\partial N}{\partial x}+v \frac{\partial N}{\partial y}=\frac{\sigma}{\rho j} \frac{\partial^{2} N}{\partial y^{2}}-\frac{\kappa}{\rho j}\left(2 N+\frac{\partial u}{\partial y}\right), \\
u \frac{\partial T}{\partial x}+v \frac{\partial T}{\partial y}=\frac{\kappa^{*}}{\rho c_{p}} \frac{\partial^{2} T}{\partial y^{2}},
\end{array}
$$

with the boundary conditions:

$$
\begin{gathered}
u=-U_{w}=-a e^{\frac{x}{L}}, \quad v=v_{w}=-v_{0} e^{\frac{x}{2 L}}, \\
N=-n \frac{\partial u}{\partial y}, T=T_{w}=T_{\infty}+T_{0} e^{\frac{x}{2 L}} \text { at } \quad y=0, \\
u \rightarrow 0, \quad N \rightarrow 0, \quad T \rightarrow T_{\infty} \text { as } y \rightarrow \infty,
\end{gathered}
$$

where $\mathrm{u}$ and $\mathrm{v}$ are the fluid velocity in $x$ - and $y$-directions respectively, $v=\mu / \rho$ is the kinematic viscosity of the fluid with $\mu$ as the dynamic viscosity and $\rho$ as the density of the fluid, $\kappa$ is the vortex viscosity of the fluid, $N$ is the microrotation, $T$ is the temperature of the fluid, $\kappa^{*}$ is the thermal conductivity of the fluid, $c_{p}$ is the specific heat, $v_{w}$ is the variable suction velocity with $v_{0}$ as constant, $L$ is the reference length, $T_{w}$ is the temperature at the sheet, $T_{0}$ is the rate of temperature increment along the sheet and $T_{\infty}$ is the ambient fluid temperature. The boundary condition has $n(0 \leq n \leq 1)$ as a constant with the case $n=0$ denotes strong concentration, $n=0.5$ denotes weak concentration and $n=1$ is for turbulent boundary layer flow. According to Aurangzaib et. al., (2016), $\sigma$ is the spin gradient viscosity with

$$
\sigma=\left(\mu+\frac{\kappa}{2}\right) j=\mu\left(1+\frac{K}{2}\right) j
$$

where $j=2 L v e^{-\frac{x}{L}} / a$ is the microinertia per unit mass and $K=\kappa / \mu$ is the material parameter.

In this problem, the physical quantities of interest are the dimensionless skin friction coefficient, couple stress and Nusselt number, given by:

$$
C_{f} R e_{x}^{\frac{1}{2}} \sqrt{\frac{2 L}{x}}=(1+(1-n) K) f^{\prime \prime}(0),
$$

$$
M_{x} R e_{x}=\left(1+\frac{K}{2}\right) h^{\prime}(0),
$$

and

$$
N u_{x} R e_{x}^{-\frac{1}{2}} \sqrt{\frac{2 L}{x}}=-\theta^{\prime}(0),
$$

respectively, with local Reynolds number $R e_{x}=x U_{w} / v$. This problem has been solved by Aurangzaib et al. (2016) and the solutions are found to be of dual solutions.

Therefore, stability analysis will be performed to the solutions. Firstly, the problem is considered to be unsteady where the flow is dependent of time. The continuity equation (1) holds and the governing equations for the unsteady problem are:

$$
\begin{gathered}
\frac{\partial u}{\partial t}+u \frac{\partial u}{\partial x}+v \frac{\partial u}{\partial y}=\left(v+\frac{\kappa}{\rho}\right) \frac{\partial^{2} u}{\partial y^{2}}+\frac{\kappa}{\rho} \frac{\partial N}{\partial y} \\
\frac{\partial N}{\partial t}+u \frac{\partial N}{\partial x}+v \frac{\partial N}{\partial y}=\frac{\sigma}{\rho j} \frac{\partial^{2} N}{\partial y^{2}}-\frac{\kappa}{\rho j}\left(2 N+\frac{\partial u}{\partial y}\right) \\
\frac{\partial T}{\partial t}+u \frac{\partial T}{\partial x}+v \frac{\partial T}{\partial y}=\frac{\kappa^{*}}{\rho c_{p}} \frac{\partial^{2} T}{\partial y^{2}}
\end{gathered}
$$

where $t$ is the time. The similarity transformations are given as:

$$
\begin{gathered}
N=\left(\frac{a}{2 L v}\right) \sqrt{2 L v a} e^{\frac{3 x}{2 L}} h(\eta, \tau), \quad \theta(\eta, \tau)=\frac{T-T_{\infty}}{T_{w}-T_{\infty}}, \\
\psi=\sqrt{2 L v a} e^{\frac{x}{2 L}} f(\eta, \tau), \quad \eta=\sqrt{\frac{a}{2 L v}} e^{\frac{x}{2 L} y,} \\
\tau=\frac{a}{2 L} e^{\frac{x}{L} t},
\end{gathered}
$$

where $\tau$ is the dimensionless time variable and $\psi$ is the stream function with

$$
u=\frac{\partial \psi}{\partial y}, v=-\frac{\partial \psi}{\partial x}
$$

This results to

$$
\begin{gathered}
u=a e^{\frac{x}{L}} \frac{\partial}{\partial \eta} f(\eta, \tau), \\
v=-\left[\sqrt{\frac{a v}{2 L}} e^{\frac{x}{2 L}} f(\eta, \tau)+\frac{a}{2 L} y e^{\frac{x}{L}} \frac{\partial}{\partial \eta} f(\eta, \tau)\right. \\
\left.+t\left(\frac{a}{L}\right) \sqrt{\frac{a v}{2 L}} e^{\frac{3 x}{2 L}} \frac{\partial}{\partial \tau} f(\eta, \tau)\right] .
\end{gathered}
$$

Equations (10) and (11) are then substituted into Eqs. (7)-(9) to obtain the following ordinary differential equations:

$$
\begin{gathered}
(1+K) \frac{\partial^{3} f}{\partial \eta^{3}}+f \frac{\partial^{2} f}{\partial \eta^{2}}-2\left(\frac{\partial f}{\partial \eta}\right)^{2}-2 \tau\left(\frac{\partial f}{\partial \eta} \frac{\partial^{2} f}{\partial \eta \partial \tau}-\frac{\partial f}{\partial \tau} \frac{\partial^{2} f}{\partial \eta^{2}}\right) \\
-\frac{\partial^{2} f}{\partial \eta \partial \tau}+K \frac{\partial h}{\partial \eta}=0
\end{gathered}
$$




$$
\begin{gathered}
\left(1+\frac{K}{2}\right) \frac{\partial^{2} h}{\partial \eta^{2}}+f \frac{\partial h}{\partial \eta}-3 h \frac{\partial f}{\partial \eta}-K\left(2 h+\frac{\partial^{2} f}{\partial \eta^{2}}\right)-\frac{\partial h}{\partial \tau} \\
-2 \tau\left(\frac{\partial f}{\partial \eta} \frac{\partial h}{\partial \tau}-\frac{\partial f}{\partial \tau} \frac{\partial h}{\partial \eta}\right)=0
\end{gathered}
$$

$$
\begin{gathered}
\frac{1}{\operatorname{Pr}} \frac{\partial^{2} \theta}{\partial \eta^{2}}+f \frac{\partial \theta}{\partial \eta}-\theta \frac{\partial f}{\partial \eta}-\frac{\partial \theta}{\partial \tau}-2 \tau\left(\frac{\partial \theta}{\partial \tau} \frac{\partial f}{\partial \eta}-\frac{\partial f}{\partial \tau} \frac{\partial \theta}{\partial \eta}\right) \\
=0,
\end{gathered}
$$

with $\operatorname{Pr}=\mu c_{p} / \kappa^{*}$ as the Prandtl number. The boundary conditions are:

$$
\begin{gathered}
f(0, \tau)=S=v_{0} \sqrt{\frac{2 L}{a v}}, f^{\prime}(0, \tau)=-1, \\
h(0, \tau)=-n f^{\prime \prime}(0, \tau), \quad \theta(0, \tau)=1, \\
f^{\prime}(\infty, \tau) \rightarrow 0, \quad h(\infty, \tau) \rightarrow 0, \quad \theta(\infty, \tau) \rightarrow 0,
\end{gathered}
$$

where $S>0$ is the suction parameter.

According to Weidman et. al., (2006), the stability of the steady flow solution $f(\eta)=f_{0}(\eta), h(\eta)=h_{0}(\eta)$ and $\theta(\eta)=\theta_{0}(\eta)$ that satisfies the boundary value problem in (1)-(6) can be tested by introducing the following equations:

$$
\begin{gathered}
f(\eta, \tau)=f_{0}(\eta)+e^{-\gamma \tau} F(\eta, \tau), \\
h(\eta, \tau)=h_{0}(\eta)+e^{-\gamma \tau} H(\eta, \tau), \\
\theta(\eta, \tau)=\theta_{0}(\eta)+e^{-\gamma \tau} G(\eta, \tau),
\end{gathered}
$$

such that $\gamma$ is the unknown eigenvalue and $F(\eta, \tau), H(\eta, \tau)$ and $G(\eta, \tau)$ are small relative to $f_{0}(\eta), h_{0}(\eta)$ and $\theta_{0}(\eta)$. An infinite set of eigenvalues $\left(\gamma_{1}<\gamma_{2}<\gamma_{3}<\cdots\right)$ will be produced by the unsteady problem in (12)-(15) (2016). If the smallest eigenvalue, $\gamma_{1}$ is less than zero $\left(\gamma_{1}<0\right)$, the flow is not stable due to an initial growth of disturbance, whereas if $\gamma_{1}>0$, there is initial decay of disturbance for stable flow.

Equation (16) is substituted into Eqs. (12)-(15). Then, the following linearized problem is obtained:

$$
\begin{gathered}
(1+K) \frac{\partial^{3} F}{\partial \eta^{3}}+f_{0} \frac{\partial^{2} F}{\partial \eta^{2}}+(1-2 \tau \gamma) F \frac{\partial^{2} f_{0}}{\partial \eta^{2}}+(2 \tau \gamma-4) \frac{\partial f_{0}}{\partial \eta} \frac{\partial F}{\partial \eta} \\
-\left(2 \tau \frac{\partial f_{0}}{\partial \eta}+1\right) \frac{\partial^{2} F}{\partial \eta \partial \tau}+\gamma \frac{\partial F}{\partial \eta}+2 \tau \frac{\partial F}{\partial \tau} \frac{\partial^{2} f_{0}}{\partial \eta^{2}} \\
+K \frac{\partial H}{\partial \eta}=0, \quad(17) \\
\left(1+\frac{K}{2}\right) \frac{\partial^{2} H}{\partial \eta^{2}}+f_{0} \frac{\partial H}{\partial \eta}+\left(F-2 \tau \gamma F+2 \tau \frac{\partial F}{\partial \tau}\right) \frac{\partial h_{0}}{\partial \eta}-3 h_{0} \frac{\partial F}{\partial \eta} \\
-(3-2 \tau \gamma) H \frac{\partial f_{0}}{\partial \eta}-K \frac{\partial^{2} F}{\partial \eta^{2}} \\
-\left(1+2 \tau \frac{\partial f_{0}}{\partial \eta}\right) \frac{\partial H}{\partial \tau}-H(2 K-\gamma) \\
=0
\end{gathered}
$$

$$
\begin{aligned}
\frac{1}{\operatorname{Pr}} \frac{\partial^{2} G}{\partial \eta^{2}}+f_{0} \frac{\partial G}{\partial \eta}+ & \left(F+2 \tau \frac{\partial F}{\partial \tau}-2 \tau \gamma F\right) \frac{\partial \theta_{0}}{\partial \eta}-(1-2 \tau \gamma) G \frac{\partial f_{0}}{\partial \eta} \\
& -\theta_{0} \frac{\partial F}{\partial \eta}-\left(1+2 \tau \frac{\partial f_{0}}{\partial \eta}\right) \frac{\partial G}{\partial \tau}+\gamma G \\
& =0,
\end{aligned}
$$

with the boundary conditions:

$$
\begin{gathered}
F(0, \tau)=0, \frac{\partial}{\partial \eta} F(0, \tau)=0, \\
H(0, \tau)=-n \frac{\partial^{2}}{\partial \eta^{2}} F(0, \tau), \quad G(0, \tau)=0, \\
\frac{\partial}{\partial \eta} F(\eta, \tau) \rightarrow 0, H(\eta, \tau) \rightarrow 0, G(\eta, \tau) \rightarrow 0 \text { as } \eta \rightarrow \infty .
\end{gathered}
$$

Next, $\tau$ is set to $0(\tau=0)$, so that the solutions of $f(\eta)=$ $f_{0}(\eta), h(\eta)=h_{0}(\eta)$ and $\theta(\eta)=\theta_{0}(\eta)$ can be obtained. Then, $F=F_{0}(\eta), H(\eta)=H_{0}(\eta)$ and $G(\eta)=G_{0}(\eta)$ in Eqs. (17)-(19) identify the initial growth or decay of the solution (16). The resulting linear eigenvalue problem are:

$$
\begin{aligned}
&(1+K) F_{0}^{\prime \prime \prime}+f_{0} F_{0}^{\prime \prime}+f_{0}^{\prime \prime} F_{0}+\left(\gamma-4 f_{0}^{\prime}\right) F_{0}^{\prime} \\
&+K H_{0}^{\prime}=0,(21) \\
&\left(1+\frac{K}{2}\right) H_{0}^{\prime \prime}+f_{0} H_{0}^{\prime}+F_{0} h_{0}^{\prime}-K F_{0}^{\prime \prime}-3 h_{0} F_{0}^{\prime} \\
&-H_{0}\left(3 f_{0}^{\prime}+2 K-\gamma\right)=0,(22) \\
& \frac{1}{P r} G_{0}^{\prime \prime}+f_{0} G_{0}^{\prime}+F_{0} \theta_{0}^{\prime}-G_{0} f_{0}^{\prime}-\theta_{0} F_{0}^{\prime} \\
&+\gamma G_{0}=0,(23)
\end{aligned}
$$

with the boundary conditions:

$$
\begin{gathered}
F_{0}(0)=0, \quad F_{0}^{\prime}(0)=0, \\
H_{0}(0)=-n F_{0}^{\prime \prime}(0), G_{0}(0)=0, \\
F_{0}^{\prime}(\eta) \rightarrow 0, \quad H_{0}(\eta) \rightarrow 0, \quad G_{0}(\eta) \rightarrow 0 \text { as } \eta \rightarrow \infty .
\end{gathered}
$$

As stated by Harris et al. (2009), the range of eigenvalues can be determined by relaxing the boundary condition either at $F_{0}(\eta), H_{0}(\eta)$ or $G_{0}(\eta)$. In this study, the boundary condition $F_{0}^{\prime}(\eta) \rightarrow 0$ as $\eta \rightarrow \infty$ is selected to relax. Equations (21)-(23) are then solved along the following boundary conditions:

$$
\begin{gathered}
F_{0}(0)=0, \quad F_{0}^{\prime}(0)=0, \quad F_{0}^{\prime \prime}(0)=1, \\
H_{0}(0)=-n F_{0}^{\prime \prime}(0), \quad G_{0}(0)=0, \\
H_{0}(\eta) \rightarrow 0, \quad G_{0}(\eta) \rightarrow 0 \text { as } \eta \rightarrow \infty .
\end{gathered}
$$

\section{RESULTS AND DISCUSSION}

The present study is an extension to the study done by Aurangzaib et. al., (2016). In the previous study, dual solutions were obtained. Figure 1 shows the velocity profile for the problem studied by Aurangzaib et. al., (2016). It shows clearly that dual solutions exist in the problem. It can 
be seen in Fig. 2, the dual solutions exist when the value of suction parameter $S$ is greater than the value at the critical point $S_{c}$, one solution at $S$ equal to $S_{c}$ and no solution when $S$ is less than $S_{c}$. Therefore, stability analysis is performed to determine the stability of the solutions.

A solver in MATLAB called the bvp4c is used to solve Eqs. (21)-(23) along the new boundary conditions (25). The values of $\gamma_{1}$ for several values of $S$ are computed and shown in Table 1. In the table, the first solution has values of $\gamma_{1}>$ 0 , while the second solution has values of $\gamma_{1}<0$. As stated by Awaludin et al. (2016), the values of $\gamma_{1}>0$ indicates thestable solution, while $\gamma_{1}<0$ implies theunstable solution. Therefore, based on the results in Table 1 , it can be concluded that the stable solution is the first solution while the second solution is unstable. The first solution of this problem is said to be physically meaningful and more realizable in practice.

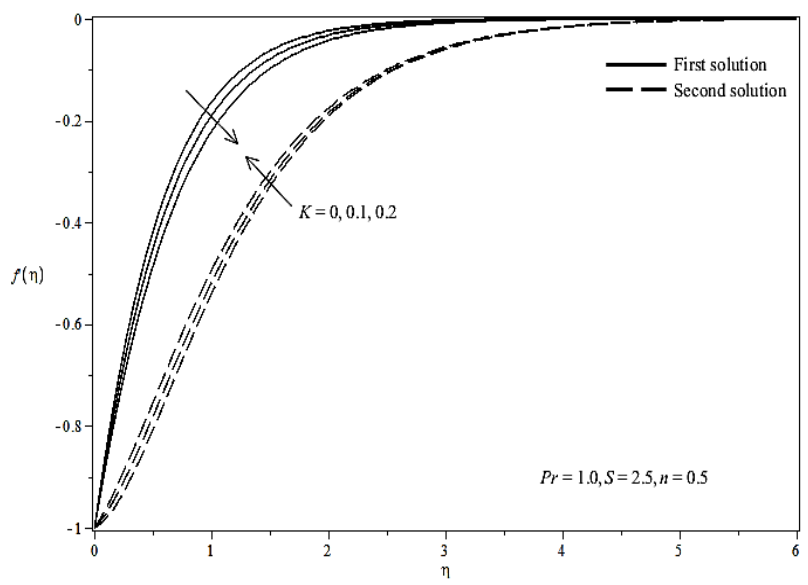

Figure 1. Velocity profile $f^{\prime}(\eta)$ for different values of material parameter $K$.

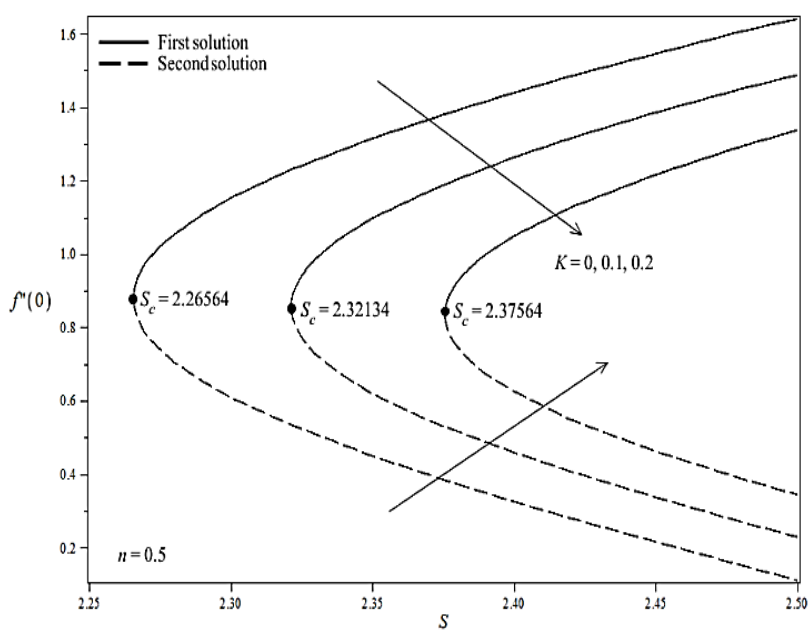

Figure 2. Skin friction coefficient $f^{\prime \prime}(0)$ with $S$ and various values of $K$.
Table 1. Smallest eigenvalues $\gamma_{1}$ for different values of $S$ when $\operatorname{Pr}=1.0, n=0.5$.

\begin{tabular}{cccc}
\hline$K$ & $S$ & $1^{\text {st }}$ solution & $2^{\text {nd }}$ solution \\
\hline 0 & 2.266 & 0.03477 & -0.03471 \\
& 2.267 & 0.06752 & -0.06728 \\
& 2.268 & 0.08898 & -0.08857 \\
0.1 & 2.322 & 0.04660 & -0.04648 \\
& 2.323 & 0.07387 & -0.07358 \\
& 2.324 & 0.09353 & -0.09308 \\
& & & \\
0.2 & 2.376 & 0.03434 & -0.03428 \\
& 2.377 & 0.06636 & -0.06613 \\
& 2.378 & 0.08739 & -0.08698 \\
\hline
\end{tabular}

\section{CONCLUSION}

Stability analysis is performed to the dual solutions obtained in the study by Aurangzaib et. al., (2016). The problem is considered as unsteady problem with flow dependent of time. Then, the computations of the smallest eigenvalue $\gamma_{1}$ are done by using the bvp4c solver. It is found that the first solution has positive values of $\gamma_{1}\left(\gamma_{1}>\right.$ 0 ), while the second solution has negative values of $\gamma_{1}\left(\gamma_{1}<0\right)$. Thus, the first solution is said to be stable and more meaningful than the second solution.

\section{ACKNOWLEDGEMENT}

The authors gratefully acknowledge financial support from the Universiti Putra Malaysia in the form of Putra Grant [957060o]. 


\section{REFERENCES}

Aurangzaib, Bhattacharyya, K., Shafie, S. 2016, Effect of partial slip on an unsteady MHD mixed convection stagnation-point flow of a micropolar fluid towards a permeable shrinking sheet. Alexandria Engineering Journal, 55(2), 1285-1293.

Aurangzaib, Uddin, M. S., Bhattacharyya, K., Shafie, S. 2016, Micropolar fluid flow and heat

transfer over an exponentially permeable shrinking sheet. Propulsion and Power Research, 5(4), 310-317.

Awaludin, I. S. and Weidman, P. D. and Ishak, A. 2016, Stability analysis of stagnation-point flow over a stretching/shrinking sheet. AIP Advances, 6(4).

Bhattacharyya, K., Mukhopadhyay, S., Layek, G. C., Pop, I. 2012, Effects of thermal radiation on micropolar fluid flow and heat transfer over a porous shrinking sheet. International Journal of Heat and Mass Transfer, 55(11), 2945-2952.

Eringen, A. C. 1966, Theory of micropolar fluids. Journal of Mathematics and Mechanics, 1-18.

Harris, S. D., Ingham, D. B., Pop, I. 2009, Mixed convection boundary-layer flow near the stagnation point on a vertical surface in a porous medium: Brinkman model with slip. Transport in Porous Media, 77(2), 267-285.

Khan, Z. H., Qasim, M., Ishfaq, N., Khan, W. A. 2017, Dual solutions of MHD boundary layer flow of a micropolar fluid with weak concentration over a stretching/shrinking sheet. Communications in Theoretical Physics, 67(4), 449.

Merkin, J. H. 1986, On dual solutions occurring in mixed convection in a porous medium. Journal of Engineering Mathematics, 20(2), 171-179.

Roşca, N. C., Pop, I. 2014, Boundary layer flow past a permeable shrinking sheet in a micropolar fluid with a second order slip flow model. European Journal of Mechanics-B/Fluids, 48, 115-122.

Saleh, S. H. M., Arifin, N. M., Nazar, R., Pop, I. 2017 , Unsteady micropolar fluid over a permeable curved stretching shrinking surface. Mathematical Problems in Engineering, 1-13.

Sandeep, N., Sulochana, C. 2015, Dual solutions for unsteady mixed convection flow of MHD micropolar fluid over a stretching/shrinking sheet with non-uniform heat source/sink. Engineering Science and Technology, an International Journal, 18(4), 738-745.

Sharma, R., Ishak, A., Pop, I. 2016, Stagnation point flow of a micropolar fluid over a stretching/shrinking sheet with second-order velocity slip. Journal of Aerospace Engineering, 29(5), 04016025.

Weidman, P. D. and Kubitschek, D. G. and Davis, A. M. J. 2006, The effect of transpiration on self-similar boundary layer flow over moving surfaces. International Journal of Engineering Science, 44(11), 730-737.

Yacob, N. A., Ishak, A. 2012, Micropolar fluid flow over a shrinking sheet. Meccanica, 47(2), 293-299.

Zaimi, K., Ishak, A. 2014, Stagnation-point flow and heat transfer over a nonlinearly stretching/shrinking sheet in a micropolar fluid. Abstract and Applied Analysis, 2014, 1-6. 